\title{
Projet qualité: histoire d'une certification d'un hôpital
}

\author{
R. Schroeder
}

Le projet de qualité avec une certification APEQ d'un hôpital vaudois avec une organisation complexe (trois sites et trois missions) est décrit. Le travail accompli, les avantages et les limites d'un tel projet sont présentés et discutés d'un point de vue médicale.
_ plus de 800 questionnaires patients traités;

- une centaine de plaintes traitées;

- plus de 400 documents identifiés, classés, répertoriés;

- 4 enquêtes de satisfaction dont la première destinée aux collaborateurs;

- 2 brochures d'information tirées chacune à 750 exemplaires;

- 61 collaborateurs ayant suivi une formation de répondant qualité.

- nombreux projets en attente, qui ont dû être réalisés pour répondre aux exigences des référentiels: système de gestion du matériel et des infrastructures, gestion des moyens de contention, généralisation du consentement éclairé, mise à jour de tous les cahiers des charges et entretiens d'appréciation, concepts douleur, nutrition, soin, accompagnement, animation ...

- Orbe (à $10 \mathrm{~km})$ : réadaptation, soins palliatifs, policlinique, hébergement;

- La Vallée (Le Sentier à 30 km): centre médicochirurgical, chirurgie, médecine interne et hébergement.

L'hôpital dessert prioritairement les districts vaudois de Cossonay, d'Orbe et de La Vallée ce qui correspond à une population d'environ 46000 habitants.

\section{Calendrier et travaux accomplis}

Le projet a été dirigé par un responsable qualité supervisé par un comité de pilotage sous la responsabilité du directeur général. Après une préparation de cinq mois, le projet commençait en décembre 2001 avec un pré-audit en juin 2003 et

1 PEQ: agence de promotion et d'évaluation de la qualité.

2 ISO: International Organisation for Standardisation.

Correspondance: Dr Rolf Schroeder resHO

CH-1318 Pompaples

Tél. 0218665111

Fax 0218665122

E-mail: rolf.schroeder@resho.ch l'audit de certification en février 2004.

Les travaux accomplis pendant ces deux ans et deux mois en chiffre:

- 600 sous projets menés à bien;

- plus de 100 groupes de travail;

- quelques 500 fiches de signalement de non conformités, incidents critiques, proposition d'amélioration;

- 15 audits réalisés à l'interne;

- 90 indicateurs répertoriés;

\section{Participation des médecins}

Le début du projet coïncidait avec la nomination d'un directeur médical à $20 \%$, poste nouvellement créé. Il était membre du Comité de pilotage et consacrait une partie de son activité à ce projet. La charge de travail pour les autres médecins était ainsi réduite. Certains participaient à des groupes de travail, d'autres étaient sollicités pour élaborer des documents. Lors du pré-audit et de l'audit, plusieurs médecins-chefs, médecins agréés et médecins-assistants étaient consultés. Les documents importants étaient sélectionnés par le responsable du projet et le directeur médical et mis à leur disposition.

\section{Bénéfice pour I'hôpital et pour les patients}

L'intérêt principal d'un tel projet de certification n'est pas médical mais concerne la direction et l'administration. Les instruments et la technique de gestion et les contrôles de qualité ont pu être introduits impliquant tous les services.

L'impact sur la sécurité des patients et la qualité de travail de tous les jours sur le terrain est 
difficile à évaluer. Le système $\mathrm{SPEQ}^{3}$ définit les procédures mais ne tient que peu compte du résultat médical. Avec quelques exceptions, nous n'apprenons rien sur nos techniques opératoires, sur les complications et la prise en charge des patients en médecine interne et dans les autres services.

Le bénéfice est donc indirect. Les exigences de qualité donnaient au directeur médical un cadre pour organiser le service médical avec 70 médecins (chefs, agréés, consultants et médecins-assistants). L'organisation et les cahiers des charges des différents services pouvaient être clarifiés ainsi que le flux d'information et la communication entre les services. Un plan de formation pour les médecins-assistants a été créé. La présence d'un responsable qualité aidé d'un secrétariat et d'un comité de pilotage ont facilité la réalisation rapide de projets prévus depuis un certain temps et qui devaient être introduits indépendamment de la démarche qualité: deux projets étaient ainsi prioritaires: l'introduction du consentement éclairé et le signalement d'incidents critiques.

Une responsable de sécurité a été nommée et un concept de sécurité et de gestion des risques élaboré. Ceci permettait d'évaluer les risques dans tous les services à l'aide de check-lists et de mettre en place des actions de prévention.

Certains indicateurs de qualité introduits intéressaient en particulier les médecins: les infections nosocomiales, les chutes de patients, les escarres, la satisfaction des patients et des collaborateurs, le délai de l'envoi de lettres de sortie, l'évaluation des stages par les médecins-assistants.

La Direction et le corps médical ont été sensibilisés aux droits des patients et la démarche a permis d'accélérer l'intégration de ces droits dans l'organisation.

Les procédures pour traiter les réclamations ont été créées et permettent une réaction rapide et efficace.

Pour s'assurer d'un bénéfice pour les patients, le travail de qualité doit continuer après la certification. Un exemple: au moment de la certification, un concept de prise en charge de la douleur avait été rédigé et tous les médecins-assistants étaient équipés d'une échelle de la douleur. Il est évident que ce n'était pas suffisant. Dès la certification réalisée, il était nécessaire de préparer un plan de sensibilisation et de formation à ce nouveau concept. Des objectifs prioritaires et limités doivent être identifiés pour pouvoir changer les habitudes et pour appliquer les procédures dans la pratique, au lit du malade. La certification en elle-même ne fait pas tout. Les signalements d'incidents critiques et de non-conformités gardent toute leur importance et les procédures doivent être régulièrement révisées.

\section{Conclusion}

Un projet qualité implique un travail considérable et indispensable pour réévaluer, redéfinir l'organisation, les structures, et les procédures de l'hôpital. Le projet donne à la direction des instruments de technique de gestion et de contrôles de qualité importants. Bien que le projet ne tienne que peu compte du résultat médical, il y a un bénéfice indirect pour les patients en clarifiant les structures et l'organisation médicales, par la formation des médecins-assistants, par l'introduction d'indicateurs de qualité, par l'élaboration d'un concept de sécurité et de gestion des risques et par le signalement d'incidents critiques. Il est vrai que la plupart des erreurs sont humaines et ne sont pas dues à une structure et à une organisation mais la méthode prévoit des cahiers des charges précis, une évaluation régulière et une formation continue de tout le personnel travaillant dans l'hôpital.

Les évènements récents (erreurs dans le cadre d'une transplantation du cœur) ont montré l'énorme intérêt de la population et des médias pour la sécurité des patients dans nos hôpitaux. La certification diminue la probabilité d'erreurs dans certains domaines, mais ne nous protège pas entièrement de celles-ci. Elle nous permet en revanche de prouver avoir fait le maximum pour les éviter. Nous devons cet effort à nos patients qui nous accordent leur confiance.

Il est d'autant plus regrettable que les responsables de la santé publique du canton et les caisses maladies n'ont pas ou peu réagi à notre certification démontrant ainsi leur désintérêt pour les efforts de qualité.
SPEQ: système de promotion
et d'évaluation de la qualité. 